\title{
The band-edge light emission from the metal-oxide-silicon tunneling diode on (1 110$)$ substrates
}

\author{
S.T. Chang, K.F. Chen, C.R. Shie, C.W. Liu *,1, Miin-Jang Chen, \\ Ching-Fuh Lin ${ }^{*, 2}$
}

Department of Electrical Engineering, National Taiwan University, 1, Section 4, Roosevelt Road, Taipei 10617, Taiwan, ROC

Received 9 September 2001; received in revised form 4 December 2001; accepted 2 January 2002

\begin{abstract}
The band-edge electroluminescence at room temperature from metal-oxide-silicon tunneling diodes on (1 110$)$ substrates was observed. Both the transverse acoustic and the transverse optical phonon lines are necessary to fit emission spectra. A comprehensive picture composed of localized holes, phonons and interface roughness is given to describe the radiation process. The picture can be used to explain the enhanced electroluminescence intensity, as compared to photoluminescence, and can be used to understand the substrate orientation effect on electroluminescence intensity. (c) 2002 Elsevier Science Ltd. All rights reserved.
\end{abstract}

Keywords: MOS diode; Electroluminescence; Electron-hole plasma recombination; Tunneling

\section{Introduction}

The band-edge light emission from metal-oxide-silicon (MOS) diodes attracts a great attention not only due to technological importance [1,2], but also due to the understanding of the radiation process itself $[3,4]$. An electron-hole-plasma (EHP) recombination model was given to fit the line shape of the electroluminescence, and the band-edge luminescence nature was confirmed [3] on the $\left(\begin{array}{lll}1 & 0 & 0\end{array}\right)$ substrate. The temperature dependence study of electroluminescence (EL) spectra on (100) samples suggested that the reduction of bandgap extracted from the emission spectra was primarily due to the transverse optical (TO) phonon as well as the surface band bending [4]. To further probe the radiation process, the MOS tunneling diodes were fabricated on p-type (110) Si substrates. Note that the natural cleavage plane on

\footnotetext{
${ }^{*}$ Corresponding author. Tel.: +886-2-23635251x515; fax: +886-2-23638247.

E-mail address: chee@cc.ee.ntu.edu.tw (C.W. Liu).

${ }^{1}$ Also with Graduate Institute of Electronic Engineering.

${ }^{2}$ Also with Institute of Electro-Optical Engineering.
}

(1 10$) \mathrm{Si}$ is perpendicular to the substrate, and hence a cavity structure can be easily fabricated. The similar band-edge emission spectra were observed and can be also fit by the EHP recombination model for the devices on the (110) substrate. Previous study of $\mathrm{Si} / \mathrm{SiGe} / \mathrm{Si}$ quantum wells on (110) Si substrates [5] showed that the no-phonon (NP) peak in the (110) substrate is relatively intense, as compared to $\left(\begin{array}{ll}1 & 0\end{array}\right)$ substrate probably due to the larger conduction band offset on the (110) substrate than that on the $\left(\begin{array}{ll}10 & 0\end{array}\right)$ substrate. Although the emission intensity of the MOS diodes depends on a variety of factors such as defects in the oxide, defects in the $\mathrm{SiO}_{2} / \mathrm{Si}$ interface, and etc., we observed that the EL intensity on p-type (1 110$)$ substrates seems stronger than that on p-type $(100)$ substrates. A picture will be given to explain this observation.

\section{Device fabrication}

The ultrathin gate oxide of the MOS tunneling diode used in this study is grown by rapid thermal oxidation (RTO) at the $1000{ }^{\circ} \mathrm{C}$. The gas flows are $500 \mathrm{sccm}$ nitrogen and $500 \mathrm{sccm}$ oxygen at the pressure of $250 \mathrm{mbar}$. 
Before oxidation, the sample was cleaned by a HF dip. An in situ hydrogen bake at $900{ }^{\circ} \mathrm{C}$ for 2 min was performed before the growth of the RTO. The temperature was measured by a pyrometer with a close loop control. After the growth of the ultrathin oxide, the sample was in situ annealed subsequently in hydrogen and in nitrogen for 10 min each at $900{ }^{\circ} \mathrm{C}$. The nominal thickness of the oxide is about $2.4 \mathrm{~nm}$, measured by ellipsometry. The resistivity of the $100 \mathrm{~mm}$ p-type (1 110$)$ wafers is 1 $10 \Omega \mathrm{cm}$. The NMOS (p-substrate) diodes had Al gate electrodes with circular areas defined by photolithography.

\section{Results and discussion}

Fig. 1 shows current-voltage $(I-V)$ curve of an $\mathrm{Al} / 2.4$ $\mathrm{nm}$ oxide/p-silicon NMOS diode with a circular area of $3 \times 10^{-4} \mathrm{~cm}^{2}$ at room temperature. For the $\mathrm{Al}$ gate under negative bias, the electron in the $\mathrm{Al}$ tunnels to the Si substrate through the thin oxide. For the magnitude of the negative gate bias less than $1 \mathrm{~V}$, the electron tunnels to the interface states in the oxide/Si interface [6], and cannot contribute to the light emission process. For the magnitude of the negative gate bias larger than 1 $\mathrm{V}$, the electron starts to tunnel into the conduction band of Si substrates. The negative gate bias also attracts the hole in the $\mathrm{Si} / \mathrm{SiO}_{2}$ interface to form an accumulation region. The hole in the Si valence band becomes localized along the growth direction and its momentum has a spread in the reciprocal space along the growth direction. The tunneling electrons in the Si conduction band can recombine with the holes in the accumulation region, and emit light if the momentum can be conserved by the phonon scattering, roughness scattering, and etc. Note that there is a kink at $\sim-1 \mathrm{~V}$ in the $I-V$ curve of NMOS device, indicating the transition of these two current-transport mechanisms. Note that the current at

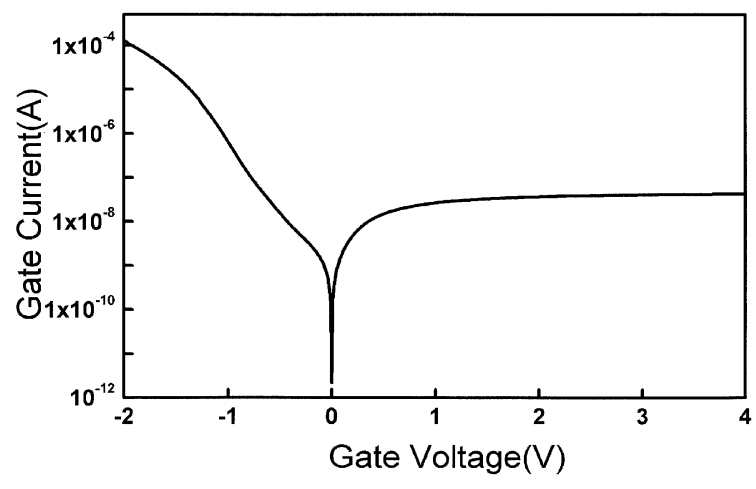

Fig. 1. The current-voltage characteristics of the NMOS diode on the p-type (1 110$)$ substrate.

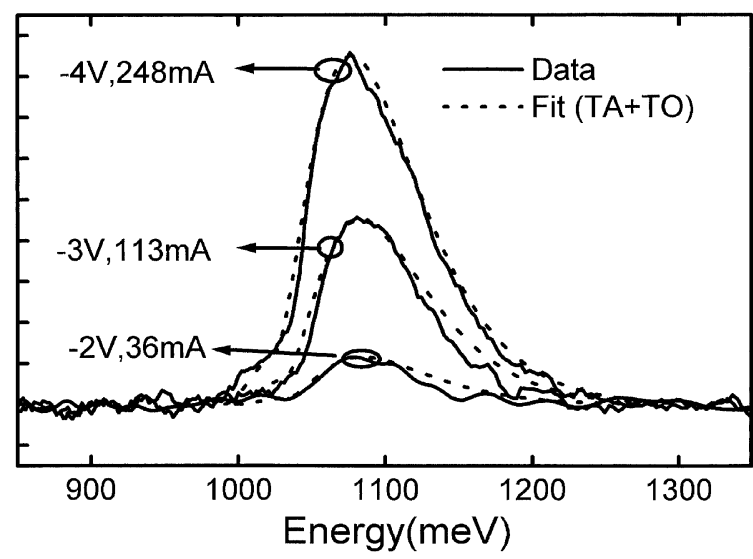

Fig. 2. The EL spectra of a (1 110$)$ NMOS diode. The simulation curves have both the TA and TO lines.

the positive gate bias is originated from the generation of minority electrons through the interface states [7]. $\mathrm{We}$, therefore, perform the EL measurement at the magnitude of the negative gate bias larger than $1 \mathrm{~V}$. Fig. 2 shows the EL spectra of the MOS diode with the size of $4 \times 10^{-2} \mathrm{~cm}^{2}$ under different gate bias. The emission intensity increases as the gate bias (gate current) increases. The width of the line shape also increases with gate current due to the band filling effect [4]. The theoretical line shapes from EHP recombination model are also shown in Fig. 2. Details of the EHP recombination model can be found in Ref. [3]. Both the transverse acoustic (TA) and transverse optical (TO) phonons can give the extra momentum needed for radiative recombination [8]. The TA phonon is $36 \mathrm{meV}$ lower in energy than the TO phonon [9]. Therefore both the TA phonon line and the TO phonon line are used to fit the experimental line shape. The relative intensity of TA phonon with respect to the TO phonon is left as an adjustable parameter and the relative ratio is around $\sim 0.2$ in our simulation. Note that if only TO phonon line is considered, the theoretical line shapes do not fit well with experimental curves in low energy part. Also note that the half width at high-energy part of the line shape is wider as compared to low energy part. When the NMOS diode is biased at negative gate voltage (Fig. 3), the electron tunnel from Al to the p-Si through the ultrathin oxide. The electron tunneling into the conduction band can relax to the band edge, and radiatively recombine with the holes in accumulation region if the momentum is conserved. The electron tunneling into the interface states cannot contribute the radiative recombination. With defects in the oxide [7], the hole in the accumulation region of $\mathrm{Si}$ can leak through the oxide. This also reduces the light emission intensity.

To address the issue of momentum conservation, we present a picture of the radiative process composed of 


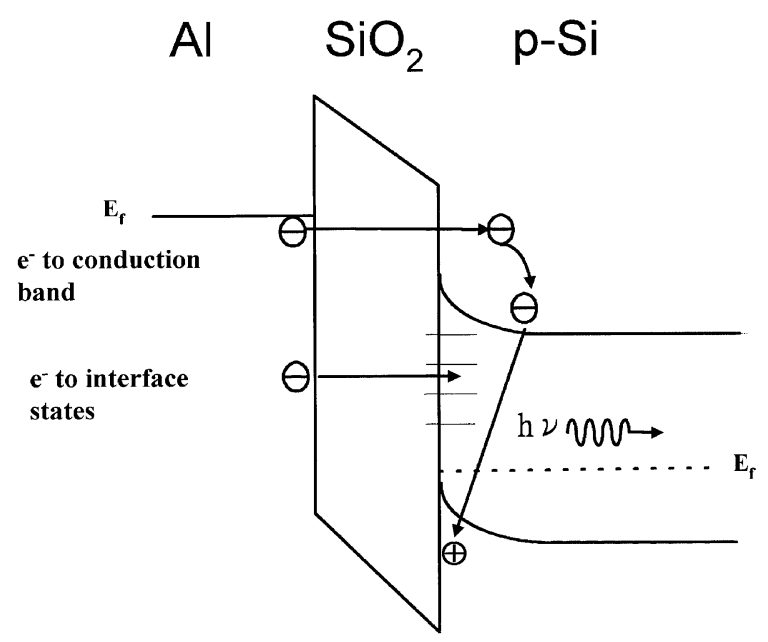

Fig. 3. Two conduction current for the NMOS tunneling diode at the negative gate bias.

phonons (both TO and TA), interface roughness, and confined holes in the accumulation region (Fig. 4). The hole confinement in the accumulation region yields a spread of momentum in the reciprocal lattice along the growth direction. Since the momentum difference between holes and electrons can be provided by the phonons [4], the phonons involved in the radiative process, do not lie in a very restricted region in the phonon dispersion curve as in the case of PL. Instead, all the phonons in the spread with the extent similar to the momentum spread of the localized holes can contribute to the radiative process. This explains that the EL intensity is much higher than PL intensity. For the (1 110$)$ NMOS devices, the spread of hole momentum along growth direction shortens the separation of hole states and electron states in the nearest four valleys. The oxide roughness also produces an additional momentum spread [10] perpendicular to the growth direction by scattering the holes at zone center to the neighborhood around the zone center. The roughness scattering was demonstrated to enhance the EL intensity as shown in Refs. [2] and [11]. Since the interface roughness is a twodimensional effect, all six valleys can be beneficial for the light emission due to this momentum spread. Note that the interface roughness can also spread the momentum around the conduction band valleys, but it is not shown in Fig. 4, since its effect is similar to the spread of hole momentum. For the $(100)$ substrate, only two conduction valleys become close to the valence edge due to the hole confinement to delocalized momentum (Fig. 4(a)). Only four valleys perpendicular to the growth direction can get close to the hole at zone center by the roughness scattering. Therefore, more electrons (valleys) are involved in the radiative process on (110) substrate (Fig. 4(b)), as compared to $\left(\begin{array}{lll}1 & 0 & 0\end{array}\right)$ substrates (Fig. 4(a)). Fig. 5

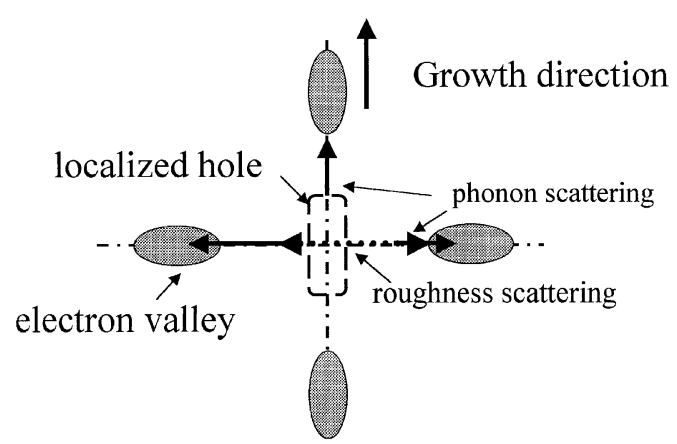

(a) (100)

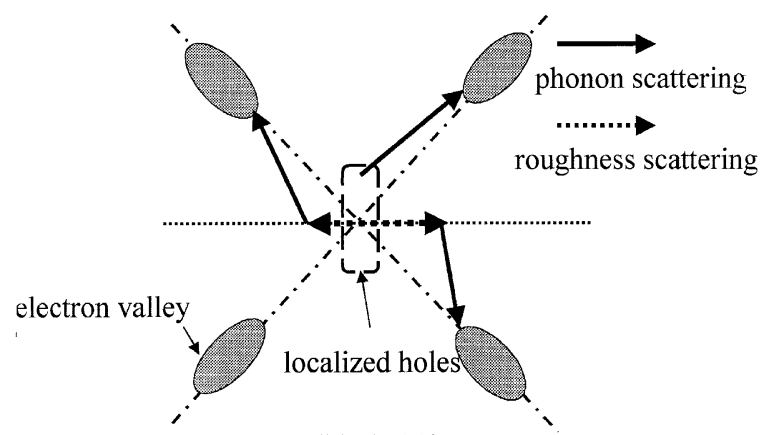

(b) (110)

Fig. 4. A proposed picture of the radiative recombination in NMOS tunneling diode. Note that the two valleys perpendicular to the paper are not shown.

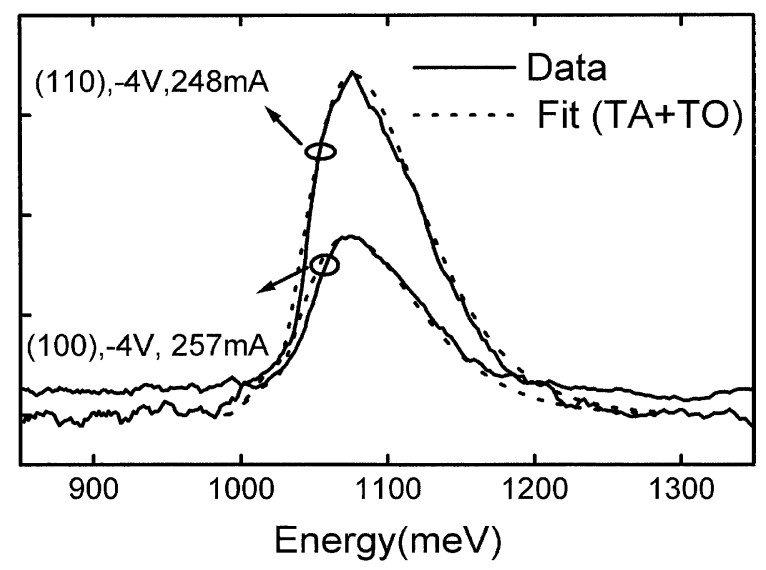

Fig. 5. A comparison of EL spectra from (1 000$)$ and (1 110$)$ substrates.

shows the EL spectra from (1 000$)$ and (1 110$)$ substrates. Although a lot of factors (Fig. 4) determine the emission efficiency, it seems that the (1 110$)$ substrate can provide more electron valleys in the radiation process, as compare to the $(100)$ substrate. 


\section{Summary}

In conclusion, the EL from NMOS on (1 110$)$ substrates was demonstrated. Both TO and TA phonon lines are necessary to fit the emission line shape using the electron-hole recombination model. A comprehensive picture of the radiation process is described. From this picture, the (110) substrate has the potential to emit more light than the $\left(\begin{array}{lll}1 & 0 & 0\end{array}\right)$ substrate.

\section{Acknowledgements}

This work is supported by National Science Council, Taiwan, ROC (89-2218-E-002-017, 89-2112-M-002-034).

\section{References}

[1] Liu CW, Lee MH, Lin CF, Lin IC, Liu WT, Lin HH. Light emission and detection by metal oxide silicon tunneling diodes. In: Proceedings of International Electron Devices Meeting (IEDM), 1999. p. 749.

[2] Liu CW, Lee MH, Chen MJ, Lin CF. Roughness-enhanced electroluminescence from metal oxide silicon tunneling diodes. IEEE Electron Device Lett 2000;21:601.

[3] Liu CW, Lee MH, Chen MJ, Lin IC, Lin CF. Roomtemperature electro-luminescence from electron-hole plas- mas in the metal oxide silicon tunneling diodes. Appl Phys Lett 2000;76(12):1516.

[4] Liu CW, Chen MJ, Lin IC, Lee MH, Lin CF. Temperature dependence of the electron-hole-plasma electroluminescence from the metal oxide silicon tunneling diodes. Appl Phys Lett 2000;77(8):1111.

[5] Liu CW, Sturm J, Lacroix YR, Thewalt MLW, Perovic DD. Growth and bandgap of strained (1 110$)$ layers on silicon substrate by rapid thermal vapor deposition. Appl Phys Lett 1994;65(1):76.

[6] Ghetti A, Sangiorgi E, Bude J, Sorsch TW, Weber G. Low voltage tunneling in ultra-thin oxides: a monitor for interface states and degradation. In: Proceedings of International Electron Devices Meeting (IEDM), 1999. p. 731.

[7] Miranda E, Sune J, Rodriguez R, Nafria M, Aymerich X, Fonseca L, Campabadal F. Soft breakdown conduction in ultrathin $(3-5 \mathrm{~nm})$ gate dielectrics. IEEE Trans Electron Device 2000;47:82.

[8] Weber J, Alonso MI. Near-band-edge photoluminescence of Si-Ge alloys. Phys Rev B 1989;40:5683.

[9] Hull R. In: Properties of crystalline silicon. UK: INSPECT; 1999. p. 160.

[10] Pirovano A, Lacaita AL, Ghidini G, Tallarida G. On the correlation between surface roughness and inversion layer mobility in Si-MOSFETs. IEEE Electron Device Lett 2000;21:34.

[11] Uehara Y, Watanabe J, Fujikawa S. Ushioda lightemission mechanism of Si-MOS tunnel junction. Phys Rev B 1995;51:2229. 